Courrier du Centre International BlaisePascal

38-39 | 2017-2018

Varia

\title{
Salomon de Tultie prophète ou la Sagesse folle des Pensées
}

Hubert Aupetit

\section{(2) OpenEdition}

1 Journals

Édition électronique

URL : https://journals.openedition.org/ccibp/1444

DOI : 10.4000/ccibp.1444

ISSN : 2493-7460

Éditeur

Centre international Blaise Pascal

Édition imprimée

Date de publication : 1 janvier 2019

Pagination : 139-158

ISBN : 978-2-84516-897-8

ISSN : 0249-6674

Référence électronique

Hubert Aupetit, «Salomon de Tultie prophète ou la Sagesse folle des Pensées », Courrier du Centre International Blaise-Pascal [En ligne], 38-39 | 2017-2018, mis en ligne le 21 juin 2021, consulté le 05 mai 2022. URL : http://journals.openedition.org/ccibp/1444; DOI : https://doi.org/10.4000/ccibp.1444

Ce document a été généré automatiquement le 5 mai 2022.

Centre international Blaise Pascal 


\title{
Salomon de Tultie prophète ou la Sagesse folle des Pensées
}

\author{
Hubert Aupetit
}

1 Louis de Montalte, Amos Dettonville, Salomon de Tultie: on a peu réfléchi au sens proprement poétique, et non seulement anecdotique, du goût pascalien pour les pseudonymes - particulièrement à ce nom très étrange, pourtant riche de sens et de connotations prophétiques, qu'est "Salomon de Tultie ", évoquant aussi bien pour le $\mathrm{XVII}^{\mathrm{e}}$ siècle la sagesse sapientiale de l'Ecclésiaste ou des Proverbes que la sagesse « folle » (stultitia) de l'Évangile.

D’illustres aînés ont lancé l'hypothèse qu'il aurait pu désigner le signataire des Pensées, sans véritablement en tirer de conséquences en termes d'interprétation. À l'heure où les éditions modernes sont remises en cause pour leur caractère de plus en plus «illisible ${ }^{1}$ », mon propos est de montrer qu'il permet de donner sens à la partie ordonnée et titrée des Pensées, que j'appelle les Pensées choisies.

3 Pascal, en définitive, aurait balancé entre deux projets : une apologie de la religion chrétienne dont nous possédons quelques grands textes, mais aucun début de mise en œuvre; une enquête sur le bonheur, dont la résolution implique le passage d'une sagesse naturelle à la sagesse divine et prophétique de l'Écriture, sous-tendue par la grande structure figurative inventée par saint Paul et confirmée par saint Augustin.

4 C'est bien cette extraordinaire enquête, pleine de rebonds et de surprises, que les éditions modernes, fondées sur l'une ou l'autre des copies de l'original pascalien, nous permettent de suivre pas à pas grâce à leurs vingt-sept premières liasses titrées, pourvu qu'on abandonne les présupposés de lecture remontant à Victor Cousin.

5 Deux rappels seront nécessaires en préambule: l'un sur la pseudonymie littéraire, l'autre sur l'ordre d'édition des Pensées. 


\section{Auctor in fabula} Proust et des études littéraires du $\mathrm{xx}^{\mathrm{e}}$ siècle?

[U]n livre est le produit d'un autre moi que celui que nous manifestons dans nos habitudes, dans la société, dans nos vices².

Est-il décidément surhumain pour une critique hypnotisée, depuis le romantisme, par la figure du génie créateur, de séparer l'homme de l'œuvre, et de considérer que tout ce que le lecteur impute au "je» qu'il lit dans un livre n'est pas systématiquement transférable au « moi » biographique de l'auteur?

plus, est-ce pécher contre l'obsession médiatique de la transparence que de risquer l'hypothèse que lorsqu'un auteur écrit sous nom d'emprunt, ce n'est pas nécessairement coquetterie ou dissimulation, mais manière de confirmer ce que les professeurs de français enseignent désormais dès les petites classes à leurs élèves dans l'étude d'un roman : ne confondez pas l'auteur et le narrateur ! L'homme qui raconte, et dont le lecteur se construit l'idée en lisant, est aussi un personnage de l'œuvre. Il peut jouer l'invisibilité ou manifester sa présence, il en est a minima le régisseur, assumant les règles et conventions d'écriture qui lui sont propres. Bardamu n'est pas Céline ${ }^{3}$, il donne au Voyage au bout de la nuit une autorité poétique qui fait barrière aux tentatives de discréditer l'ouvrage par les méfaits pamphlétaires de son auteur. Du Bellay n'est pas le poète exilé et famélique qui dit «je» dans les Regrets. Et si Montaigne a la « sottise », selon Pascal, de peindre son moi, c'est précisément parce que le « moi » que le lecteur lui prête n'est fait que de " qualités empruntées ${ }^{4}$ »- ce que confirme la critique montanienne, qui a beau jeu de souligner les contradictions et contre-vérités biographiques des Essais.

En rhétorique depuis Aristote, l'autorité fictive émanant du discours s'appelle l'ethos; elle est celle de l'avocat dont on n'interroge pas la vie privée, ni la liste des clients, ni l'inventaire des plaidoiries, avant de recevoir son éloquence. C'est elle qui parle et se construit en parlant, elle est l'auctor in fabula, fabriquée par le texte, à l'instar du lector in fabula d'Umberto Eco ${ }^{5}$. Ainsi le Pascal des Provinciales invente in fabula tant son lector, le «Provincial» ignorant des subtilités théologiques, que son auctor, l'«ami du Provincial ». Et lorsqu'il choisit de prêter à ce dernier le nom de «Louis de Montalte » dans le recueil édité des "petites lettres", il donne à l'œuvre sa dimension mystificatrice sans laquelle on ne saurait goûter pleinement son ironie ${ }^{6}$.

9 Pascal ne se contente pas de se métamorphoser, pour les besoins d'un canular littéraire, en épistolier enquêteur. Il devient avocat dans la Lettre d'un avocat au Parlement à un de ses amis touchant l'inquisition qu'on veut établir en France; docteur universitaire lorsqu'il compose avec Antoine Arnauld les Réflexions d'un docteur de Sorbonne sur l'Avis donné par l'évêque d'Alet; curé de Paris dans les Écrits éponymes; évêque dans le Projet de mandement contre l'Apologie pour les casuistes...

Le cas de «M. Pascal », dans l'Entretien avec M. de Sacy est plus retors, dont l'ethos est fabriqué par un autre que Pascal, assavoir Nicolas Fontaine. Certes, ce dernier nourrit son texte de documents authentiques écrits par Pascal, mais il invente un «M. Pascal » " extrêmement estimable en ce que, n'ayant point lu les Pères de l'Église, il avait de luimême, par la pénétration de son esprit, trouvé les mêmes vérités qu'ils avaient trouvées $^{7} »$. Or la critique biographique, en particulier Philippe Sellier, nous dit bien que Pascal, à l'époque concernée, était déjà un "augustinien consommé ${ }^{8}$ ». La

Courrier du Centre International Blaise-Pascal, 38-39 | 2017-2018 
manœuvre rhétorique est claire: il s'agit, pour l'ancien secrétaire de M. de Saci, de répartir les rôles: les auteurs profanes à «M. Pascal», saint Augustin à «M. de Saci », dans une construction littéraire à la finalité rhétorique bien précise : promouvoir une image édifiante d'un Port-Royal attirant les grands esprits de ce temps'.

11 Ce « M. Pascal » forgé par Fontaine en évoque un autre : l'auctor de l'édition princeps de Port-Royal des Pensées intitulée Pensées de M. Pascal sur la religion et sur les autres sujets. Contrairement aux éditions modernes, celle-ci attaque d'emblée son lecteur par un discours nettement polémique : "Que ceux qui combattent la Religion apprennent au moins quelle elle est avant que de la combattre ... », et le gourmande avec brutalité :

J'espère montrer ici qu'il n'y a personne raisonnable qui puisse parler de la sorte ; et j'ose même dire que jamais personne ne l'a fait. On sait assez de quelle manière agissent ceux qui sont dans cet esprit. Ils croient avoir fait de grands efforts pour s'instruire [...A]près cela, ils se vantent d'avoir cherché sans succès dans les livres et parmi les hommes. Mais, en vérité, je ne puis m'empêcher de leur dire, que cette négligence n'est pas supportable ${ }^{10}$.

Cette attitude consistant à agresser l'incroyant bille en tête ne cadre pas avec la douce tolérance manifestée par l'énonciateur de cet autre fragment célèbre des Pensées : Quand on veut reprendre avec utilité et montrer à un autre qu'il se trompe il faut observer par quel côté il envisage la chose car elle est vraie ordinairement de ce côté-là et lui avouer cette vérité, mais lui découvrir le côté par où elle est fausse ${ }^{11}$.

Pas plus qu'avec le caractère décrit par Gilberte Perier dans sa Vie de Pascal :

[Q]uand il avait à conférer avec quelques athées, il ne commençait jamais par la dispute, ni par établir les principes qu'il avait à dire, mais il voulait connaître auparavant s'ils cherchaient la vérité de tout leur cœur ; et il agissait suivant cela avec eux, ou pour les aider à trouver la lumière qu'ils n'avaient pas, s'ils la cherchaient sincèrement, ou pour les disposer à la chercher, et à en faire leur plus sérieuse occupation, avant que de les instruire, s'ils voulaient que son instruction leur fût utile ${ }^{12}$.

Que conclure, sinon que les Pensées, telles que nous les connaissons, nous mettent en présence de deux auctores contradictoires - preuve s'il en fallait encore de leur caractère heuristique, hétérogène, inachevé. Autrement dit, nous avons affaire à deux stratégies rhétoriques opposées déduites d'un même chantier : l'une combative, qu'on rattacherait sans peine au genre apologétique; l'autre cherchant à conduire avec adresse son lecteur à pousser la raison naturelle jusqu'à l'absurde, afin de rendre nécessaire l'approche scripturaire. Ce sont deux regards, deux destins possibles de l'ouvrage, à partir des brouillons que nous avons reçus.

\section{Rappel aux ordres des Pensées}

13 Chacun sait les différents assemblages possibles dont les Pensées peuvent faire l'objet. La très grande variété des éditions qui en ont été proposées, la multiplicité des numérotations, les polémiques qu'elles éveillent, en témoignent mieux que toute argumentation.

14 J'ai cité l'incipit des Pensées de Port-Royal : d'emblée le lecteur sait par un long discours qu'il a affaire à un chrétien désireux de faire connaître et de défendre sa religion vis-àvis de ceux qui la " combattent ». Il ne parle pas en homme, ni même en chrétien, mais bien en tant que chrétien.

15 Si j'entre au contraire dans les Pensées par la copie « $\mathrm{C} 1$ », je vois un premier embryon de chapitre intitulé «Vanité » où il est successivement question de visages semblables 
qui font rire, d'avoir quatre laquais, du pouvoir des mouches... Je n'ai pas l'impression d'un auteur cherchant à défendre ou à imposer une perspective religieuse, mais plutôt d'un écrivain qui, selon la définition des dictionnaires, «observe, décrit et analyse les mœurs, les passions ", autrement dit à un moraliste - de plus amateur de forme courte, privilégiant la sentence, l'aphorisme, l'écriture par fragment. Nul n'attaque, nul ne défend : nous n'entrons pas dans une apologie. L'auctor in fabula est-il même chrétien ? Assurément si nous savons qu'il s'agit de Blaise Pascal, mais constatons que ce dernier choisit de ne pas parler en tant que chrétien à son lecteur.

Il faut ici rappeler trois choses :

- (i) La question du plan des Pensées reste ouverte parce que Pascal lui-même ne l'a pas tranchée ;

-(ii) L'ordre que proposent les copies $\mathrm{C} 1$ et $\mathrm{C} 2$ ne correspond pas à un projet rhétorique défensif ;

- (iii) En tout état de cause, c'est le seul qui offre un début de réalisation éditoriale, et dont chacun s'accorde à dire qu'il a bien été conçu par Pascal - à quelques différends mineurs près entre spécialistes.

17 (i) Pascal a effectivement laissé plusieurs possibilités de plans dans sa liasse "Ordre ", dont on peut imaginer autant de réalisations effectives. Celles-ci se répartissent, en gros, selon deux types :

- - Ceux qui présupposent la religion et l'ethos d'un croyant, autrement dit faisant entrer le lecteur dans l'œuvre en se disant: «J'ai affaire à un chrétien qui veut me convaincre du bien-fondé de sa religion ». Ces plans-là, on peut sans peine les appeler «apologétiques », ainsi que les éditions qui s'en inspirent - quitte à donner à ce qualificatif le sens moderne élargi d'un discours de défense (et non seulement celui d'un discours «justifiant quelqu'un ", comme le définit Furetière) ;

- À côté de ces projets où l'auctor chrétien s'affiche d'emblée, il en est d'autres où l'on entre, on l'a vu, selon la raison ordinaire parlant de choses ordinaires de la vie - au fond de manière assez proche du modus operandi montanien, que le lecteur des Essais n'a aucune peine à retrouver dans ces premières dizaines de pages, parfois au mot et à la phrase près. Ces plans-là, c'est faire violence au texte que de les qualifier d' " apologétiques ", sauf à tout confondre et à refuser de voir la différence fondamentale entre faire défendre sa religion ou sa sensibilité religieuse par un chrétien, ou faire évoquer la religion par quelqu'un qui ne se présente pas a priori comme un de ses défenseurs. Quand Pascal veut écrire comme un curé, on l'a vu, il signe "curés de Paris », et cela donne les Écrits des curés de Paris. Quand il veut écrire comme l'ignorant qu'il n'est pas en théologie, il signe Louis de Montalte et ce sont les Provinciales. Quand il sait qu'il va donner de lui l'image d'un mathématicien quelque peu vaniteux alors qu'il a renoncé à tirer gloire des mathématiques depuis plusieurs années, il crée le personnage d'Amos Dettonville - non sans humour si l'on songe qu'Amos est le prophète biblique qui répond à ses contradicteurs $(A \operatorname{mos} 7,14)$ :

Je ne suis ni prophète, ni fils de prophète ; je suis berger, et je cultive les sycomores.

(ii) et (iii). Parmi tous les plans envisageables pour les Pensées, il en est un qui mérite notre attention parce qu'il est le seul dont nous possédons un début de réalisation objective, donc scientifique selon les canons de la philologie moderne : c'est l'ordre des copies, qu'adoptent avec plus ou moins d'aménagements ou d'ajouts les éditions publiées des Pensées depuis Louis Lafuma (1949). Il regroupe vingt-sept liasses ou dossiers ordonnés selon une table des matières qui les répertorie en deux colonnes en leur donnant des titres; on n'est pas sûr qu'elle reproduise un original autographe, 
mais l'ordre qu'elle propose correspond à un classement des dossiers tel qu'on les a trouvés sur le bureau de Pascal après sa mort.

La table en diptyque soigneusement figurée des liasses titrées (ici copie $\mathrm{C} 1$ )

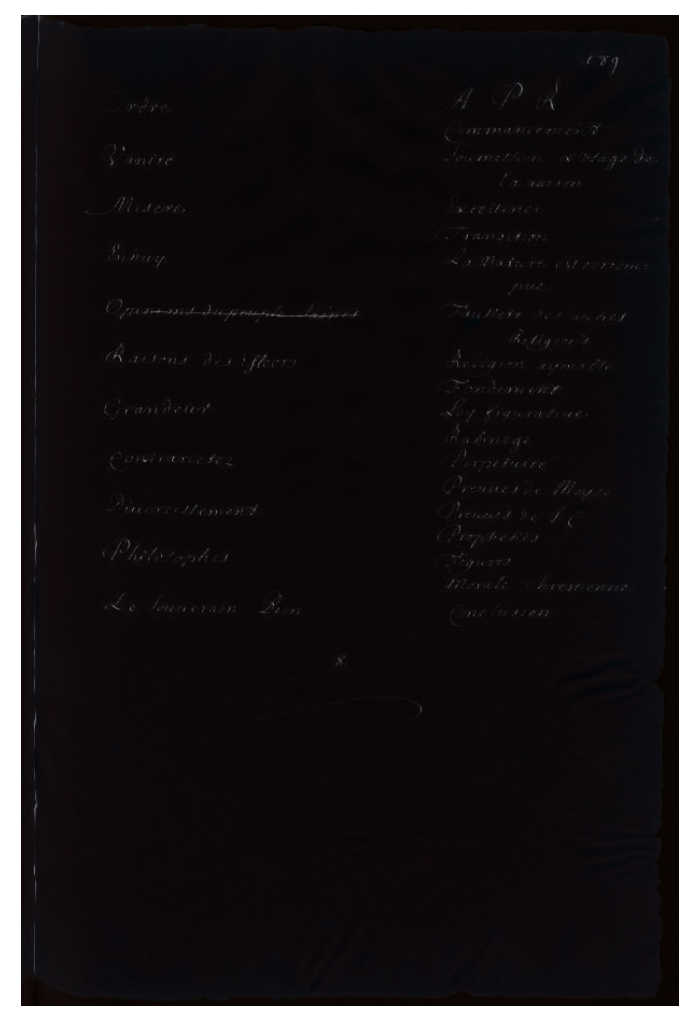

19 La même table, à un détail près, apparaît dans les deux copies. Elle répartit dans une première colonne des chapitres à thème profane, et tasse dans une seconde les chapitres à thème religieux, de manière apparemment figurée, comme s'il s'agissait de bien séparer en deux parties l'une et l'autre matières - en fait, de passer à l'enquête religieuse après épuisement de l'enquête profane ${ }^{13}$. Je les mentionnerai désormais sous le nom de " première » ou de " seconde colonne », aussi bien pour désigner les colonnes de titre de la table que leur réalisation dans les copies, et j'appelle Pensées choisies la séquence ordonnée des vingt-sept liasses titrées que reproduisent les copies en tête de l'ouvrage. C'est le seul support éditorial objectif des Pensées.

Tous les papiers qui n'appartiennent pas aux Pensées choisies sont alors à considérer comme appartenant à des Dossiers mis en réserve, agencés diversement selon les sources, ce qui explique les importantes variations entre les éditions de type Sellier (faites sur C2) et celles de type Lafuma (faites sur C1) - ainsi que la disparate déconcertante de leur numérotation aux yeux d'un néophyte qui se demande à quelle cabale il convient d'adhérer afin d'entrer en Pascalie.

21 Reste que pour la partie choisie, les écarts sont mineurs - au problème près d'un premier dossier (ou liasse) non titré de la copie C2 équivoquement nommé «liassetable» et placé en tête d'ouvrage par Philippe Sellier, que Lafuma repousse à la "Section I" des Dossiers mis en réserve sous l'intitulé "dossier de travail ». Cette divergence sur les interprétations des deux copies, ainsi que l'absence de référence à cette «liasse-table» dans la... table, me conduisent à écarter ce dossier de ce que j'appelle l'édition objective. 
22 En résumé, il convient de séparer les Pensées en deux parties : une première séquence textuelle appelée Pensées choisies ${ }^{14}$ répondant à un ordre conçu par Pascal; une seconde masse de morceaux ou pensées mis en réserve, faite de tout le reste, classés en séries dont on ignore l'agencement, et qu'on pourrait intituler, si l'on tient à l'appellation ${ }^{15}$, Projet d'apologie.

Ajoutons trois dernières remarques :

24 - Tous les grands discours qui ont pu faire ranger les Pensées dans le genre apologétique appartiennent aux Dossiers mis en réserve, dont bien sûr le "Qu'ils apprennent au moins... » faisant l'incipit de l'édition de Port-Royal, et que Philippe Sellier intitule "Lettre pour porter à rechercher Dieu ", le morceau dit du pari, les préfaces, etc. Philippe Sellier propose d'ailleurs un cas d'école intéressant : après sa première édition philologique des Pensées selon la copie C2 en 1976, il a entrepris et publié chez Presses Pocket en 2003 une édition revendiquée comme apologétique, sans numérotation, bouleversant l'ordre de la copie C2 : significativement, il doit puiser dans les Dossiers mis en réserve les textes qu'il place en ouverture, dont l'incipit de Port-Royal.

- À l'opposé, pour qui lirait les Pensées choisies pour la première fois sans appareil préfacier, sans savoir qui est Pascal, ce qui frappe, c'est qu'on entre en matière comme dans une œuvre de philosophe ou de moraliste profane, qui réfléchit «selon les lumières naturelles » pendant une dizaine de chapitres avant de faire entrer le lecteur dans ce que les éditeurs apologétiques appellent généralement «la religion chrétienne ", appellation qu'on peut aussi discuter: il est fort peu question de la religion en tant qu'institution dans les Pensées choisies (pas plus d'ailleurs que dans l'ensemble des Pensées).

26 - Enfin ce plan consistant à parler d'abord des choses ordinaires de la vie avant d'interroger la religion, essentiellement pour ses fondements scripturaires, on ne peut le ranger dans les plans apologétiques, sauf à tout embrouiller : on ne reçoit pas de la même façon, d'une part, le discours autoritaire d'un homme se revendiquant chrétien et reprochant à son lecteur de ne pas l'être ; d'autre part, celui d'un auctor développant son sujet empiriquement avant de conclure à la nécessité de quitter l'approche profane pour faire aboutir son enquête sur le bonheur. Ce sont deux projets rhétoriques différents, porteurs de principes de persuasion différents, reposant sur deux ethos discoureurs différents. L'histoire des éditions des Pensées montre que l'un et l'autre sont possibles à reconstituer à partir du chantier inachevé. Il paraît donc vain (ou demihabile ?) de polémiquer en affirmant que l'un ou l'autre est le projet qu'eût mené à bien l'auteur s'il avait vécu plus longtemps...

\section{L'hypothèse Salomon de Tultie}

27 Je ne suis pas le premier à émettre l'idée que ce pseudonyme de Salomon de Tultie a pu servir à Pascal pour envisager d'autoriser l'ouvrage qu'il avait mis en chantier. Louis Marin en faisait « la crypte du vrai nom » de Pascal :

Salomon de Tultie, le roi sage de Stultitia ; Roi de sagesse et de folie, double nom : nom propre d'un Roi, le plus sage des rois, nom commun de l'insensé. Amos (Dettonville) Louis (de Montalte) : le prophète (Amos) - Roi (Louis) de la sagesse qui est folie ; le prophète roi de charité, Jésus-Christ. 
Le style si reconnaissable de Louis Marin ne permet pas toutefois de reconnaître si pour lui le pseudonyme ("à la fois cryptonyme et pseudonyme puisqu'il est cryptonyme de pseudonymes ») est simple coquetterie mondaine :

L'honnête homme évite de se nommer et même d'employer les mots de «je » et de «moi ».

Ou artifice d'une « rhétorique du secret » propre à un art de persuader évangélique :

Le secret est ainsi ce qu'il s'agit de croire et non de savoir. Il n'est pas de l'ordre du discours mais de l'ordre de l'indication. Toutefois ce n'est ni « je » ni « Moi » qui montre mais l'Autre qui se montre dans les simulations dissimulantes de mon nom, l'Autre, un Toi qui se désigne dans le lieu où «je » parle et où, parlant j'indique Toi d'où je parle en parlant dans mon nom. Il ne s'agit pas de savoir, ni de convaincre, il ne s'agit que de persuader. Toutefois je ne persuade pas un autre, ce n'est pas moi qui le fais croire, c'est toi par ce que je dis, parce que tu fais aimer. « Il faut aimer pour connaître ; on n'entre dans la vérité que par la charité $»^{16}$.

Ainsi parlait l'oracle.

Jean Mesnard, avec la clarté qu'on lui connaît, avait lui aussi émis la possibilité d'imputer les Pensées à Salomon, mais en ne s'intéressant qu'à la psychologie de la création pascalienne :

Les Pensées fournissent une autre anagramme de Louis de Montalte, Salomon de Tultie, sous lequel songeait sans doute à se dissimuler l'auteur de l'Apologie. On remarquera que les deux derniers prénoms, Amos et Salomon, offrent un même caractère biblique très significatif. C'est dire que, pour Pascal, il existait entre les trois ouvrages une unité plus profonde que celle d'un même auteur: celle d'un même dessein ${ }^{17}$.

Michel Le Guern est, de tous, celui qui prit le plus nettement position sur la question, en tentant même une répartition des fragments des Pensées sous les autorités respectives de Pascal et de son double :

Il ne faut pas se hâter d'affirmer l'identité de «la manière d'écrire [...] de Salomon de Tultie » avec le style des Pensées. Que de nombreux fragments aient été destinés à être intégrés, sous forme plus ou moins corrigée, dans l'apologie, c'est incontestable; mais ce n'est qu'une partie des Pensées, sans doute moins du tiers. Les Pensées conservent trois types de textes: ceux où c'est Salomon de Tultie qui s'exprime, ceux où Pascal écrit pour lui-même (méditations personnelles), et ceux qu'il écrit à l'intention de Salomon de Tultie, ou de Louis de Montalte, ou d'autres encore de ses doubles (notes préparatoires) ${ }^{18}$.

Je retiens donc des différentes propositions de Louis Marin, Jean Mesnard et Michel Le Guern qu'une partie des Pensées a été écrite par un Pascal s'identifiant à Salomon de Tultie, c'est-à-dire régissant son écriture sous l'autorité d'un personnage fictif au patronyme parodiquement, et même calembourdesquement, biblique. Je fais un pas de plus en imputant à Salomon les Pensées choisies.

Une première difficulté surgit : si l'on admet que Pascal voulait jouer sur la connotation biblique du pseudonyme, comment se fait-il que la première partie de l'ouvrage ignore la Bible?

\section{Naissance de Salomon}

Commençons par rappeler qu'un fragment, un seul, de l'ensemble de l'œuvre de Pascal mentionne Salomon de Tultie. Il s'agit d'un hapax, mais d'un hapax riche de 
signification, appartenant aux Dossiers mis en réserve - et pour cause, on va le voir -, collé dans le Recueil Original des fragments autographes au verso du feuillet 443.

L'écriture n'est pas de Pascal mais de Gilberte Périer qui en a reçu, pense-t-on, la dictée.

La manière d'écrire d'Épictète, de Montaigne et de Salomon de Tultie est la plus d'usage, qui s'insinue le mieux, qui demeure plus dans la mémoire et qui se fait le plus citer, parce qu'elle est toute composée de pensées nées sur les entretiens ordinaires de la vie; comme quand on parlera de la commune erreur qui est dans le monde que la lune est cause de tout, on ne manquera jamais de dire que Salomon de Tultie dit que lorsqu'on ne sait pas la vérité d'une chose il est bon qu'il y ait une erreur commune, etc. qui est la pensée de l'autre côté ci-dessus ${ }^{19}$.

Le fragment mentionnant Salomon de Tultie.

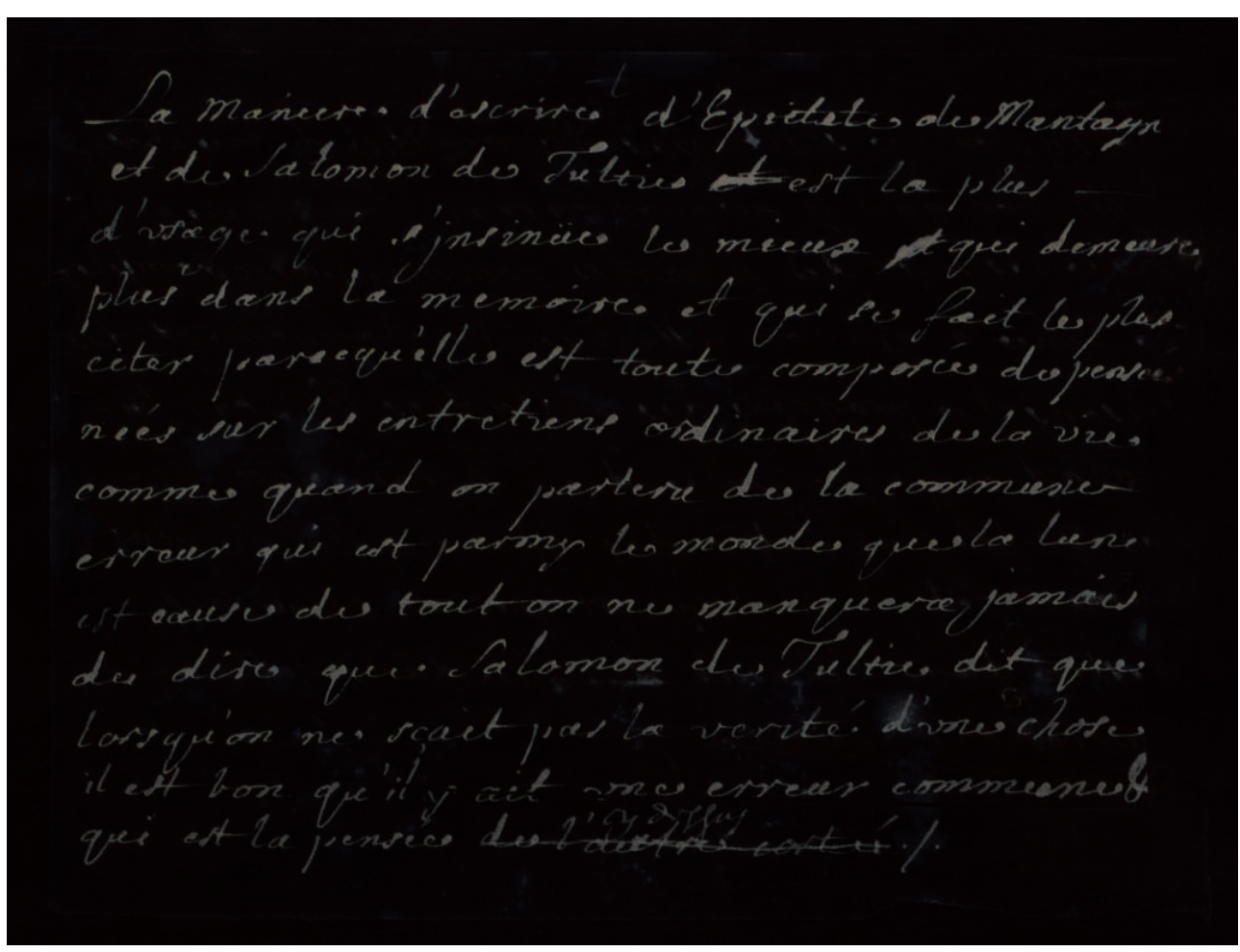

Notons que Gilberte avait d'abord écrit « de l'autre côté », et qu'effectivement, de l'autre côté du feuillet original, donc au recto, figure un autre fragment évoquant le thème partagé de «l'erreur commune »:

Lorsqu'on ne sait pas la vérité d'une chose il est bon qu'il y ait une erreur commune qui fixe l'esprit des hommes comme par exemple la lune à qui on attribue le changement des saisons, le progrès des maladies, etc., car la maladie principale de l'homme est la curiosité inquiète des choses qu'il ne peut savoir et il ne lui est pas si mauvais d'être dans l'erreur que dans cette curiosité inutile.

Lafuma différencie ce dernier fragment du précédent, numéroté 745, en lui donnant le numéro 744 - quand Sellier fusionne les deux dans le même numéro (Sel. 618). Le choix de Lafuma paraît plus pertinent car les deux critiques n'obéissent pas au même principe d'énonciation: dans le Laf. 745, Pascal parlait de Salomon de Tultie à la troisième personne; dans le 744, on va le confirmer, c'est Salomon lui-même qui s'exprime.

Notons que Pascal désigne en passant dans le Laf. 745 la forme littéraire qui caractérise à ses yeux l'écriture de Salomon de Tultie: c'est la "pensée », mot dont d'autres 
fragments montrent qu'il a bien conscience de l'acception stylistique, formelle. Ainsi le Laf. 696 / Sel. 575 joue sur les deux sens du terme :

J'aimerais autant qu'on me dise que je me suis servi des mots anciens. Et comme si les mêmes pensées ne formaient pas un autre corps de discours par une disposition différente, aussi bien que les mêmes mots forment d'autres pensées par leur différente disposition.

Peut-on imaginer que Port-Royal s'est inspiré de cette caractérisation du «corps de discours » salomonite pour donner à l'œuvre de Pascal le titre qui lui est resté jusqu'à aujourd'hui ? Notons que même si sa vogue littéraire est tout juste à sa naissance, l'usage du terme " pensée » est déjà courant pour désigner une forme courte d'écriture, par exemple pour les ouvrages moraux des anciens : Plutarque, Marc-Aurèle depuis le $\mathrm{XVI}^{\mathrm{e}}$ siècle ; en 1657 L'esprit de Sénèque, ou Les plus belles pensées de ce grand philosophe, par M. de La Serre, ou quelques années plus tôt, parues à grand bruit, les Pensées morales du S. Bardin sur l'Ecclésiaste de Salomon, recueil de paraphrases dont l'auteur se dit " interprète de Salomon ${ }^{20}$ ".

Ce qui est sûr en tout cas, c'est que les éditeurs de Port-Royal ont lu le fragment hapax 745 avec attention puisque la "pensée de l'autre côté " a été corrigée d'une autre main en «la pensée ci-dessus» sur le manuscrit de Gilberte. Comment expliquer cette correction? Quand on consulte les copies, on voit que la pensée en question 745 a effectivement été placée au-dessus sur la même page, et non au verso de la 744, signe qu'un réviseur attentif a tenu en préparant le manuscrit pour la copie à rectifier une indication rendue trompeuse par la nouvelle mise en page. La preuve en est que le copiste, contrairement à son habitude scrupuleuse, n'a pas jugé utile de reproduire la biffure, la sachant non pascalienne et purement éditoriale ( $C 1$ p 392) :

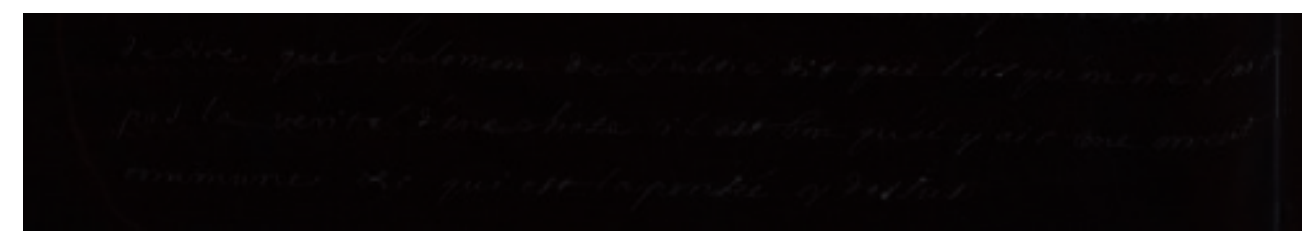

Curieusement, la plupart des éditions modernes (Lafuma, Sellier... Le Guern et l'édition électronique exceptés) maintiennent "de l'autre côté", sans doute parce qu'ils y entendent un écho au topos pascalien de la "pensée de derrière ». L'inconvénient de cette leçon fausse est majeur. Elle masque le choix par Pascal du mot "pensée », non pas pour désigner un contenu, mais bien une forme littéraire typique de "la manière d'écrire" de Salomon. Elle empêche de saisir un moment d'invention majeur où l'écrivain se sépare de son avatar salomonite. Cette opération de scissiparité se déroule en deux temps: le fragment 745 caractérise de l'extérieur l'écriture de Salomon, le Laf. 744 livre un premier échantillon de celle-ci ; Pascal est entré dans la peau de son auctor. Elle nous donne, ce faisant, un premier critère doublement précieux, puisque formel et thématique, pour distinguer comme le voulait Michel Le Guern les textes de la créature de ceux de son créateur: ce sont des "pensées", forme courte, autonome, portant sur les « entretiens ordinaires de la vie».

Cette opération par laquelle Pascal fabrique un auctor in fabula ne pouvait se faire que dans le laboratoire du projet de 1658, donc dans ce que j'appelle «les Dossiers mis en réserve». Constatons d'ailleurs que si le fragment 744 indiqué par "ci-dessus ", partie haute du Sel.618, est repris dans l'édition de Port-Royal de 1678, le Laf. 745 ne l'est pas. Les premiers lecteurs des Pensées n'ont donc jamais entendu parler de Salomon de Tultie, moins encore de cette possible autorisation d'une partie de l'œuvre, ce, pendant 
près de deux siècles. Victor Cousin lui-même n'y prête pas attention. C'est Prosper Faugère, premier éditeur post-cousinien des Pensées, qui le remarque dans son édition de 1844 sans songer à l'identifier à un avatar pascalien.

Nos recherches et celles de plusieurs érudits n'ayant pu nous procurer aucune notion sur Salomon de Tultie, nous supposons que madame Perier, de la main de laquelle ce passage se trouve écrit dans le manuscrit, aura altéré le nom de l'écrivain cité par Pascal ${ }^{21}$.

Enfin Ernest Havet, huit ans plus tard, cite dans sa première édition de 1852 la note de Faugère, et fait le premier l'hypothèse qu'il pourrait s'agir de Pascal, partant de ce que le nom est " tracé très-distinctement, et à deux fois » :

[E]n supposant que madame Perier se soit trompée, quel autre nom faudra-t-il mettre à la place? On n'en trouve aucun dans l'histoire littéraire qui convienne ici. Comment Pascal, qui semble avoir si peu lu, lisait-il un écrivain que personne ne connaît, et qu'il nomme à côté d'Épictète et de Montaigne ? On serait tenté de croire que Salomon de Tultie n'est qu'un pseudonyme, un ami de Pascal, par exemple, qui lui avait soumis quelque recueil de Pensées où Pascal avait remarqué celle qu'il cite. Ou qui sait si ce n'est pas lui-même que Pascal désigne ainsi²2.

La deuxième édition de 1855 complète la note et dévoile le mystère, dans le même art. VII, 17(bis) :

M. Frédéric Chavannes, pasteur à Amsterdam, écrivant sur Pascal dans la Revue de théologie et de philosophie chrétiennes, t. VIII (avril 1854), signale cette note [de la première édition de Havet], et en particulier la question qui la termine, et il ajoute : "Cette question nous a mis sur la voie. Le nom de Salomon de Tultie n'est autre chose que l'anagramme de Louis de Montalte. »

Ainsi ce n'est que tardivement que la postérité a pu être informée, et pas toujours clairement, de la question poétique essentielle qui se jouait dans ces deux fragments. En ignorant le fragment 745, ou en le fusionnant au 744, ou en maintenant la leçon fautive de la "pensée de l'autre côté ", les éditions des Pensées ne permettent pas de voir fonctionner le laboratoire de la création pascalienne, où le créateur tient à se distinguer nettement de sa créature auctoriale et lui prescrit un modèle d'écriture coupée, ou fragmentaire ${ }^{23}$.

\section{Personnalité de Salomon}

Qui est donc ce Salomon dans l'esprit de son inventeur? De toute évidence un pseudonyme destiné à assumer une opération littéraire dont Pascal fait mine dans le fragment 745 d'être l'un des premiers lecteurs.

41 À quel stade du chantier est-il apparu? Quelle partie du chantier devait-il endosser? Pour tenter de répondre, il convient d'approfondir l'analyse des précieuses indications poéticiennes données par l'auteur sur son auctor in fabula. C'est quelqu'un qu'il compare rien moins qu' ' à Montaigne et à Épictète, dont le style s'insinue dans la mémoire et qui se fait citer, parce que sa rédaction est toute composée de pensées nées sur les entretiens ordinaires de la vie. "

Un examen rapide de la première colonne des Pensées choisies montre qu'effectivement il n'y est question que de choses « ordinaires de la vie». On l'appelle souvent la "partie anthropologique » de l'œuvre, pour la distinguer de la seconde, « religieuse ». Le terme d'anthropologie pourrait cependant laisser croire à un discours didactique suivi sur l'homme, il n'en est rien. Ce qui caractérise ces "pensées", c'est que ce sont formellement des "pensées", organisées en fragments séparés, délibérés, rendus 
autonomes par des coups de ciseaux à partir de grandes feuilles rédigées comme l'a montré Pol Ernst. Elles se succèdent non syntaxiquement, mais parataxiquement, à l'instar des «pensées » de "l'Ecclésiaste de Salomon » publiées quelques décennies plus tôt, et bien sûr de leurs modèles sapientiaux scripturaires. Cette disposition permet en particulier de faire varier l'éclairage sur le même objet.

L'échantillon du style de Salomon de Tultie proposé par Pascal au fragment 744 est particulièrement éclairant. Il porte sur le thème, repris à Montaigne, de l'erreur commune regardée comme une bonne chose. On peut en faire la colonne vertébrale de la première partie des Pensées choisies, qui ne cesse de l'évoquer et de l'approfondir par différentes mises en perspective. La liasse "Raisons des effets", particulièrement, le ressaisit dans une structure étiologique plus fine que celle de l'erreur simplement opposée à la vérité. Car il y a toujours une vérité dans l'erreur commune, c'est qu'elle est... commune et fait donc coutume. Le peuple a certes tort de croire que les personnes de grande naissance valent mieux que lui, mais il a raison de faire comme si c'était vrai, n'en déplaise aux demi-habiles fauteurs de frondes sanglantes.

Une fois établi qu'on peut avoir en même temps raison et tort au regard des lumières naturelles, la réflexion est mûre pour assurer le basculement dans la partie scripturaire des Pensées choisies: c'est parce que les deux grandes sagesses philosophiques prétendant assurer le bonheur humain cumulent la vérité et l'erreur qu'il devient nécessaire de passer à un mode de pensée, à une sagesse, d'un autre ordre, capable de faire signifier la contradiction plutôt que de l'exclure. À une écriture régie par le principe de non-contradiction succède par nécessité une écriture fondée sur un autre principe, prophétique, que Salomon de Tultie va tirer de l'Écriture et analyser, longuement et diversement, dans la liasse «Loi figurative » (seconde colonne). C'est là toute la dynamique de basculement de ce que j'ai appelé dans un article récent "l'argument $\mathrm{APR}^{24}$ ", par lequel on passe d'une enquête à l'autre, et dont les prosopopées apparaissent comme autant de tentatives d'exposition avant un nouveau « commencement ».

\section{Les trois bonnes raisons d'attribuer les Pensées choisies à Salomon de Tultie}

On peut distinguer trois moments dans l'enquête que proposent les vingt-sept liasses titrées: les deux colonnes et leur charnière «APR». Chacun motive puissamment l'autorité de Salomon de Tultie, sage, prêtre et prophète.

(i) La première colonne/ partie des Pensées choisies contient pour l'essentiel ce que le fragment 745 présente comme «des pensées nées sur les entretiens ordinaires de la vie ». Montaigne, Épictète, ne cessent en effet de «commer », comme dit l'essai du livre I "De la force de l'imagination", c'est-à-dire de trouver l'exemple pris à l'expérience ordinaire, présente ou passée, le mieux adapté à leur discours.

Mais, dira-t-on, et Salomon?

Une... erreur commune au XVII ${ }^{\mathrm{e}}$ siècle fait du grand roi le signataire de quatre livres sapientiaux, l'Ecclésiaste, l'Ecclésiastique, la Sagesse et les Proverbes, comme le confirme M. de Saci dans la préface de ce dernier livre - qui est aussi la première traduction de l'Ancien Testament publiée par Port-Royal, en 1672. Le Livre des Proverbes est fort prisé dans le milieu port-royaliste : «c'est proprement une morale 
dont Dieu est l'auteur ", dit Saci. S'il concède quelques différences de style au Livre de la Sagesse, ce dernier les impute à un rédacteur tardif, chargé de collationner les dits du roi de Jérusalem. Et s'il accorde que l'Ecclésiastique est de Jésus Ben Sirach, il justifie par le style les opinions qui ont pu en attribuer la paternité à Salomon :

[Q]uoiqu'il soit certain que l'Ecclésiastique n'est pas de Salomon, mais de Jésus fils de Sirach, qui vivait longtemps après, on ne laissait pas, dit saint Augustin de l'attribuer à Salomon, à cause de la ressemblance du style (L. 17. de la Cité de Dieu c. 20).

Élie Faure, dans sa magnifique thèse de 1900, La Sagesse divine, insiste sur le style « ordinaire » des livres sapientiaux par opposition aux passages extraordinaires qu'on trouve dans la Bible :

Ce n'est plus guère le représentant de Iahvé, dépeint par les prophètes et l'ancienne littérature historique, avec sa mission et ses devoirs envers l'humanité, qui nous est ici présenté, mais au contraire un peuple à peu de chose près semblable aux autres peuples, ses voisins, dans sa vie laïque, civile, s'il est permis de parler ainsi quand il s'agit des Israélites. Nous sommes loin, bien loin, des rêves brillants des prophètes et de leurs élans sublimes vers la justice parfaite du royaume idéal ; les auteurs didactiques nous ramènent plus près de la terre vers les réalités ordinaires de la vie [je souligne]; çà et là quelques envolées passagères et sans consistance; du lyrisme primitif, l'esprit a presque complètement disparu, remplacé par la réflexion et l'observation des faits ; la vie simple des premiers âges a fait place aux complexités toujours croissantes des sociétés qui marchent vers la civilisation ${ }^{25}$.

Cette lecture de ceux qu'Élie Faure appelle aussi les « poètes gnomiques » de la Bible est déjà celle de Pascal qui, dans son dossier de travail, vante l'empirisme de l'auteur Salomon ${ }^{26}$ :

Salomon et Job ont le mieux connu la misère de l'homme, et en ont le mieux parlé ; l'un le plus heureux des hommes, et l'autre le plus malheureux; l'un connaissant la vanité des plaisirs par expérience, l'autre la réalité des maux.

Une note de régie sibylline de la liasse « Misère » - « Job et Salomon ${ }^{27}$ »- s'éclaire par ce fragment. Elle rappelle sans doute qu'il conviendra à l'auctor des Pensées choisies de reprendre les observations de ces deux personnages, comme il sait faire usage de celles de Montaigne et d'Épictète, afin de rapporter «l'expérience de la vanité des plaisirs » ou « la réalité des maux ».

(ii) L'analyse des textes charnière d'«APR », assurant la transition de la connaissance naturelle à une connaissance inspirée par l'Écriture, a fait l'objet de l'article cité supra. Le point à développer ici est celui dont on s'étonne en général si peu : l'outrecuidance prophétique d'un orateur qui parodie la Sagesse divine dans deux longs essais de prosopopées :

N'attendez point, ô hommes, ni vérité, ni consolation des hommes. Je suis celle qui vous ai formés et qui puis seule vous apprendre qui vous êtes....

On constate en effet, après Pierre Courcelle ${ }^{28}$ et Jean Mesnard ${ }^{29}$, que la nouveauté $\mathrm{d}^{\prime} \mathrm{APR}$ ne tient pas à son contenu, qui ne fait que reprendre la synthèse proposée par «M. Pascal » à la fin de l'Entretien:

C'est elle [la vérité de l'Évangile], qui accorde ces contrariétés par un art tout divin: unissant tout ce qu'il y a de vrai et chassant tout ce qu'il y a de faux, elle en fait une sagesse véritablement céleste, où s'accordent ces opposés qui étaient incompatibles dans les doctrines humaines ${ }^{30}$.

Toute l'originalité est énonciative : dans l'Entretien, c'est « M. Pascal » qui parle et décrit la sagesse divine; dans APR, on la fait parler. On prend souvent ces morceaux pour le texte d'une conférence prononcée à Port-Royal. J'avoue quelque réticence à imaginer Pascal contrefaisant la sagesse divine devant ses amis, dont la plupart sont prêtres et 
savants fieffés en Écriture, y compris son directeur de conscience. Il n'est certes pas rare à cette époque d'entendre en chaire des prosopopées, selon l'exemple de certains Pères de l'Église, mais les prédicateurs sont invités à en faire usage avec sobriété et parcimonie car elle risque de faire de l'orateur chrétien un comédien :

Quelque éclatante que paraisse cette Figure, comme il y a toujours de la fiction, il faut en user fort rarement et fort brièvement dans la Chaire; et l'on doit avoir un très-grand soin de bien ménager le vraisemblable ${ }^{31}$.

Pascal eût-il cabotiné sur un tel sujet? Il me paraît plus réaliste, et conforme aux habitudes chrétiennes du temps, d'une part de penser qu'il s'agit d'une forgerie biblique écrite, d'autre part de l'imputer non à Pascal, mais à son prête-nom Salomon de Tultie, dont elle représenterait une sorte de réalisation éponymique.

51 Maxime Normand, comparant les prosopopées APR à leurs modèles, note que tous les éléments qu'on y retrouve se voient réorientés vers le mythe du péché originel, alors qu'il en est «très peu question [...] dans les Livres Sapientiaux et pas du tout dans les discours de sagesse ${ }^{32}$ ». C'est que le péché originel est l'élément indispensable pour mettre en place la structure figurative et prophétique caractérisant la sagesse folle de l'Évangile, signalée par une brève note de régie d'APR suivant la seconde prosopopée : "Adam. J.- C.». Elle seule est susceptible de résoudre, dans la seconde partie de l'enquête, le problème resté en suspens dans la première.

52 (iii) C'est bien cette seconde colonne, à partir d'APR, qui complète la structure des Pensées choisies, calquée sur la grande structure figurative découverte par saint Paul dans sa première épître aux Corinthiens, reprise et développée dans celle aux Romains, et qu'il appelle le tupos.

[T]outes ces choses qui arrivaient [aux Hébreux] étaient des figures [tupos]; et elles ont été écrites pour nous servir d'instruction à nous autres, qui nous trouvons à la fin des temps ${ }^{33}$.

La figure est ce qui fait correspondre l'Ancien et le Nouveau Testament, le vieil homme et le nouvel homme et, plus précisément, chaque événement du Nouveau Testament avec un événement de l'Ancien, dont il reprend la signification en l'inversant partiellement ${ }^{34}$. Elle se rattache bien sûr à la rhétorique sémitique, puisqu'elle est binaire et parataxique ${ }^{35}$, mais elle est davantage: pour Paul et les premiers évangélistes, c'est une poétique, leur permettant de représenter par des mots ce qu'ils ont compris de cette difficile Révélation, transfigurant le monde charnel ordinaire dans la réalité extraordinaire de l'Esprit.

54 C'est bien dans cette perspective qu'on peut juxtaposer les deux colonnes des Pensées choisies. Il n'y a en effet pas de différence de thèmes entre la première et la seconde partie, cela seul qui change est la régie poétique :

55 La première colonne traite les choses ordinaires avec la raison ordinaire (régie par les principes habituels d'identité, de causalité, et de non contradiction); La seconde traite des choses ordinaires avec des règles poétiques qui leur donnent leur signification extraordinaire, à la manière exacte que pratique l'Écriture relue par saint Paul puis par saint Augustin, puis par Pascal, et qui est la manière ou régie figurative.

On notera, pour finir, que la prédication fondamentale de Paul aux Corinthiens, où apparaissent la première occurrence du tupos et la première institution de l'Eucharistie, débute par deux longues méditations sur la connaissance opposant la sagesse de Dieu à la sagesse du monde : 
Nous prêchons néanmoins la sagesse aux parfaits, non la sagesse de ce monde, ni des princes de ce monde qui se détruisent; mais nous prêchons la sagesse de Dieu, renfermée dans son mystère, cette sagesse cachée qu'il avait prédestinée et préparée avant tous les siècles pour notre gloire; que nul des princes de ce monde n'a connue, puisque, s'ils l'eussent connue, ils n'eussent jamais crucifié le Seigneur de la gloire ${ }^{36}$.

De sorte que la «sagesse » qui parle dans APR n'est pas seulement celle des modèles sapientiaux salomonites de l'Ancien Testament, mais aussi celle de Paul dans les Corinthiens et les Romains, sagesse folle - stultitia, traduit la Vulgate - qui a besoin du péché originel, donc d'Adam, pour faire signifier la foi chrétienne :

Toute la foi consiste en Jésus-Christ et en Adam et toute la morale en la concupiscence et en la grâce ${ }^{37}$.

Salomon de Tultie, dans ce troisième fragment des «Fondements de la religion chrétienne », aurait pu se contenter de résumer la foi chrétienne à Jésus-Christ : c'est bien par la figure Adam/J.-C. des Romains, annoncée dans APR, qu'il entreprend de résoudre l'enquête inconclusive sur le bonheur de la première partie : ce qui ne pouvait se résoudre au règne ordinaire de la concupiscence le peut enfin dans celui de la grâce.

\section{Conclusion}

Faut-il en revenir, comme le préconisait récemment Marc Escola, à l'édition Brunschvicg des Pensées sous prétexte de «l'illisibilité » des éditions modernes? La réponse est affirmative si l'on ne fait pas l'effort de comprendre que le chantier qu'elles nous offrent, et que nous appelons les Pensées, contient en réalité deux projets d'ouvrages : l'un constitué de morceaux, souvent longs, réunis sans principe d'édition, que l'on peut rattacher à la rhétorique apologétique ; l'autre de "pensées » choisies, soigneusement assemblées, offrant un état de réalisation suffisant pour en apprécier l'invention, la composition, la dynamique et l'originalité extraordinaires.

Ces Pensées choisies constituent la partie objective des éditions modernes. Elles se lisent comme une enquête sur le bonheur que ne peuvent résoudre les lumières naturelles, rendant nécessaire l'adoption d'un mode de pensée prophétique inspiré de l'Écriture : la typologie ou figuration, par laquelle chaque événement de l'existence ordinaire est susceptible de prendre un sens surnaturel - transfiguré.

C'est parce que chacune des parties de l'ouvrage renvoie tant à la sagesse sapientiale de l'Ancien Testament qu'à la sagesse folle du Nouveau que Salomon de Tultie, sage, prêtre et prophète, semble particulièrement désigné pour en être l'auctor in fabula.

\section{NOTES}

1. Marc Escola, «Penser, classer, avec ou sans Pascal », Acta Litt\&Arts [En ligne], p. 2, mis à jour le 30/01/2016, http://ouvroir-litt-arts.univgrenoble-alpes.fr/revues/actalittarts/ 186-penser-classer-avec-ou-sans-pascal. 
2. Marcel Proust, "La méthode de Sainte-Beuve», dans Contre Sainte-Beuve, Paris, Gallimard, « Bibliothèque de la Pléiade », 1971, p. 221-222.

3. Lequel n'est d'ailleurs pas Louis-Ferdinand Destouches, mais c'est encore une autre histoire...

4. Pascal, Pensées, Laf. 688, Sel. 567. Toutes les citations des Pensées de cet article sont prises à l'édition électronique www.penseesdepascal.fr.

5. Umberto Eco, Lector in fabula, Paris, Livre de poche, 1989.

6. Voir Laurent Thirouin, "L'èthos de Montalte dans les Provinciales ", dans "Èthos " et "pathos ». Le statut du sujet rhétorique, François Cornilliat et Richard Lockwood (dir.), Paris, Champion, 2000, p. 371-389.

7. Pascal, Entretien avec M. de Sacy, original inédit présenté par Pascale Mengotti et Jean Mesnard, Paris, Desclée de Brouwer, 1994, p. 91.

8. C'est « un augustinien consommé, qui ne se borne pas à étudier les œuvres du maître, mais analyse les commentaires qui en sont publiés » (Philippe Sellier, Pascal et saint Augustin, Paris, Albin Michel, 1995, p.15), et ce dès 1648, soit sept ans avant «l'entretien».

9. Voir Hubert Aupetit, La Nécessité biblique, thèse soutenue à l'Université de Lyon II le 28 novembre 2015, chap. 10 ou du même auteur Pascal ou la pensée figurative, à paraître chez Fayard en 2018.

10. Pensées de M. Pascal sur la religion et sur quelques autres sujets (1670 \& 1678), rééd. Georges Couton \& Jean Jehasse, Saint-Étienne, Centre interuniversitaire d'éditions et de rééditions, 1971 , p. 117.

11. Laf. 701, Sel. 579.

12. La Vie de M. Pascal ( $2^{e}$ version), dans Pascal, CEuvres complètes, éd. J. Mesnard, Paris, Desclée de Brouwer, 1964-1992 (en abrégé désormais OC), t. I, p. 622.

13. L'étude philologique la plus récente et la plus complète a été réalisée dans le plus grand détail par Gilles Proust, «Les copies des Pensées », Courrier du Centre International Blaise Pascal, $\mathrm{n}^{\circ}$ 32, Clermont-Ferrand, 2010.

14. Par «pensées choisies", j'entends simplement "choisies selon le dernier classement opéré vraisemblablement par Pascal ».

15. Sur le bien-fondé de cette appellation pour un texte du $\mathrm{XVII}^{\mathrm{e}}$ siècle, voir Vincent Carraud, «L'apologétique inactuelle, l'apologétique actuel », Communio, $\mathrm{n}^{\circ}$ XXXIX, 1-2, Paris, 2014, p. 19-32.

16. Louis Marin, "Secret, dissimulation et art de persuader chez Pascal », Revue suisse des littératures romanes, 2 (1981), p. 53-74, p. 72.

17. Pascal, OC, t. IV, p. 368.

18. Michel Le Guern, "Sur les variations d'écriture dans les Pensées », dans " Pascal a-til écrit les Pensées? », Littératures, ${ }^{\circ}$ 55, Presses Universitaires du Mirail, 2007, p. 12.

19. Laf. $745 /$ Sel. 618.

20. Paris, 1632, p. iii. Pierre Bardin fit partie de la première Académie française.

21. OC, t. I, p. 255.

22. Pensées de Pascal publiées dans leur texte authentique..., Paris, 1852, p. 108.

23. Le terme de "fragment » prête à équivoque, car il confond trois faits d'écriture : un texte court, délibérément écrit comme un fragment; un texte écrit en mode 
télégraphique; un texte plus long dont l'auteur ne donne, ou dont on ne possède, qu'une partie. On rencontre les trois types dans les Pensées. J'ai tendance à appeler «notes de régie » les deuxièmes et « morceaux » les troisièmes.

24. V. Hubert Aupetit, "Petite enquête aPRonymique ", Mélanges Dominique Descotes, à paraître chez Honoré Champion en 2018.

25. Élie Faure, La Sagesse divine, rééd. Arbre d'Or, Genève, 2007, p. 6.

26. Laf. 403/ Sel. 22.

27. Laf. 69/ Sel. 103.

28. L'Entretien de Pascal et de Sacy, ses sources et ses énigmes, Paris, Vrin, 1960.

29. OC, t. III, « Notice », p. 113.

30. Entretien, op. cit., p. 126.

31. Bretteville, L'Éloquence de la chaire et du barreau selon les principes les plus solides de la Rhétorique Sacrée et Profane, Paris, Denis Thierry, 1689, p. 251 ; cité par Servane L'Hopital, Toucher le cœur, thèse soutenue à Lyon II le 11 décembre 2015, chapitre "Jusqu'où va le prédicateur dans l'imitation? », p. 239 et suivantes.

32. Maxime Normand, Sapiential biblique et littérature morale dans la seconde moitié du dixseptième siècle en France, thèse soutenue à Paris IV le 24 mars 2007, p. 377 et suivantes.

33. 1 Corinthiens, 10,11, trad Sacy.

34. Hubert Aupetit, La Nécessité biblique, op. cit., ch. 12 et Pascal ou la pensée figurative, op. cit.

35. Voir l'article de Roland Meynet dans le même volume.

36. 1Cor 2, 6-8 (trad . Sacy).

37. Laf. 226/ Sel. 258.

INDEX

Mots-clés : pseudonymes, Pensées, sagesse

Keywords : pseudonyms, Pensées, wisdom

\section{AUTEUR}

\section{HUBERT AUPETIT}

Ancien professeur de littérature au Lycée Louis-Le-Grand, Paris 